\title{
TRADICIONALISMO Y MODERNISMO EN LA FILOSOFIA DEL DERECHO DEL SIGLO XVIII ESPAÑOL
}

La actual historiografía jurídica española nos muestra al pensamiento español sobre el derecho como siempre fiel a su inspiración medieval. Que España haya sido y sea militantemente católica la hace aparecer como la sucesora de la cristiandad medieval en oposición con el mundo de la Europa moderna, hija de la reforma protestante y laica. Aparentemente, en lo que concierne al pensamiento jurídico, la tarea de mostrarnos el tradicionalismo español es sorprendentemente fácil: la escolástica del siglo de oro (XVI y XVII), cuya importancia para el derecho nadie pone en duda, es la artífice del renacimiento de Santo Tomás y de la contrarreforma. En los siglos XIX y XX, España ha ofrecido un campo especialmente fecundo para el pensamiento jurídico neotomista. Si una corriente importante de la época como el krausismo busca su fuente de inspiración en otras doctrinas basta con excluirlo, llamándolo heterodoxo, lo que a la vez significa no católico y no español. El siglo XVIII, hasta ahora insuficientemente estudiado y calificado corrientemente como siglo de decadencia, pues en él "se perdió en gran parte la tradición nacional y (...) las corrientes extranjeras tuvieron solamente atenuada resonancia" (1), ofrece la excelente oportunidad de mostrar su tradicionalismo y así completar el esquema que hemos mencionado antes. Las dos obras españolas más recientes en la materia, "El pensamiento político del despotismo ilustrado" de SANCHEZ AGESTA (1953) y "Las ideas jurídicas en la España del siglo XVIII" de PUY MUÑOZ (1962), intentan esta demostración. En una llamarada de entusiasmo este último, por ejemplo, llega a afirmar en el comienzo de su libro que si alguna nota

1 Recasens Siches: Estudios de Filosofía del Derecho, en Del Vecchio-Recasens: Filosofía del Derecho, UTEHA, México, 1946. T. II, p. 321. puede caracterizar la comprensión del fenómeno juridico de la época es la permanencia de tradición, puesto que tanto en la consideración filosófica como en la consideración científica del derecho, se vive sobre una tradición que se remonta a la época de la Ley de las XII tablas (sic) (2).

Este intento nos parece lleno de enormes riesgos. En primer lugar, el esquema del cual parten y que a la vez pretenden demostrar nos parece sujeto a revisión. No puede aceptarse sin examen la idea de una época moderna sin lazos con la Edad Media ni la de una España heredera universal del Medievo. No puede aceptarse como artículo de fe que exista una perfecta continuidad entre el pensamiento de los teólogos españoles del siglo de oro y el de los neotomistas de los siglos XIX y XX, por una parte, y el de Santo Tomás por la otra. En segundo lugar nos parece grave desconocer el profundo interés que suscitó en la España del XVIII el pensamiento de la Europa de la época; esta apertura de España a Europa nos parece clave para comprender no sólo el pensamiento jurídico del XVIII y XIX, incluido el neotomismo, sino también la vida (3) y particularmente la vida jurídica y política española de esos siglos. Dada la indole de este artículo no podremos entrar en la discusión de los supuestos del esquema corriente de la historia del pensamiento jurídico español, sino que nos limitaremos al estudio de la actitud de los filósofos juristas españoles del siglo XVIII frente a las doctrinas europeas de la época (I) y al análisis de sus tesis (II).

2 Puy Muñoz: Las ideas jurídicas en la España del siglo XVIII. Granada, 1962.

3 V. por ejemplo el libro de HELMANN E. Trasmundo de Goya, en el cual explica los Caprichos de Goya a partir de la crítica social realizada por la élite ilustrada. 
Advirtamos por último que el estudio lo hacemos a través de tres autores: Andrés PIQUER (1711-1772), Joaquín MARIN $Y$ MENDOZA (1725-1782) y Xavier PEREZ Y LOPEZ (1736-1792). PIQUER, célebre médico y uno de los pensadores más importantes de su época en España, ha sido estudiado sobre todo en su obra Philosophia moral para la juventud española (1755, obra que conoció varias ediciones en el siglo). El y PEREZ Y LOPEZ son citados por Menéndez y Pelayo (Historia de los Heterodoxos españoles) como impugnadores del enciclopedismo y representantes de la literatura apologética. PEREZ Y LOPEZ fue un jurista importante, canonista, autor de un giganteso Teatro de la Legislación Universal de España e Indias (Madrid, 1791), enciclopedia jurídica por orden alfabético y cronológico en 28 tomos, autor igualmente de un Discurso sobre la honra y deshonra legal (1781) y de los Principios del orden esencial de la Naturaleza (1785), obra ésta que concierne directamente a la filosofía jurídica y que ha sido en consecuencia el objeto de nuestro estudio. MARIN Y MENDOZA fue el primer titular de la cátedra de Derecho Natural y de Gentes, editor de Heineccio y autor de una breve Historia del Derecho Natural y de Gentes (1776), además de obras sobre materias diversas (Itinerario que conilene el modo de hacer ccn utilidad los viajes a cortes extranjeras - 1759 - Historia de la Milicia Española - 1776-) El y PEREZ Y LOPEZ son estudiados por Sánchez Agesta como críticos de la filosofía racionalista y moderna. Se trataria, pues, de tres autores representantes del pensamiento tradicionalisła, ortodoxo (4).

\section{LOS FILOSOFOS JURISTAS ESPAÑOLES Y LA FILOSOFIA MODERNA DEL DERECHO.}

La imagen de España completamente apegada al pensamiento medieval es sin duda una ilusión. La actitud de los progresistas dieciochescos que lamentaban el atraso de su patria y que en consecuencia la veian con

4 En nuestras investigaciones hemos encontrado otro autor García Noriega (n. en Sevilla ¿-?) Autor de un discurso sobre las Qualidades que debe tener el parto en physico y civil para que se diga vital (1796) y de una Nueva Idea del Derecho Natural (1786), libro que, desgraciadamente, ha permanecido hasta ahora inaccesible para nosotros. Hemos consultado, pero hemos dejado conscientemente de lado, obras como las Cartas al Conde de Lerena de Compomanes o las Cinco cartas a Jovellanos sobre los obstáculos que la naturaleza, la opinión y las leyes oponen a la felicidad humana (1783) de Cabarrus por razones que explicamos al final de este trabajo. desesperación sumida en la más negra barbarie medieval, e igualmente la ambición de los tradicionalistas de presentarnos una España siempre fiel al cristianismo más ortodoxo, han sido los constituyentes de esa ilusión. Mostrar, como lo pretendemos en esta parte, que los filósofos juristas como sus demás contemporáneos españoles, se interesaron y aun admiraron el pensamiento europeo de su época, no equivale mostrar que ellos se hayan incorporado a ese movimiento, pero es sin duda un índice de que en alguna medida fueron marcados por su huella.

Conviene advertir que a comienzos del período que analizamos no es la doctrina jurídica o política sino la introducción en España de la ciencia nueva, que conoce un importante desarrollo en el resto de Europa, lo que preocupa a los mejores espiritus (5). Ya en 1697 se funda en Sevilla la "Sociedad Regia de Medicina y demás Ciencias" cuya finalidad principal era la de propagar los nuevos métodos científicos (6). En el mismo sentido debe interpretarse la vasta y multifacética obra del padre FElJOO y la de otros autores, generalmente médicos y que no gozan de celebridad, como Francisco SUAREZ, SOLANO MARTIN M. Hacia la mitad del siglo Andrés PIQUER, de quien nos ocuparemos menos superficialmente, logra una verdadera codificación del nuevo espiritu científico en sus obras "Lógica Moderna" (Valencia, 1947) y "Física Moderna Racional y Experimental" (Valencia, 1745). Este interés por la ciencia no es indiferente para la filosofía, y particularmente para la filosofía jurídica; sin embargo, no es fácil determinar cuál es el sentido exacto de su influencia. Podría pensarse que la ciencia constituye una nueva fe difícilmente conciliable con la fe religiosa y que la excelencia de los mejores espíritus como Feijóo y Jovellanos consiste en haber logrado una síntesis entre la orientación del pensamiento del siglo y su fe religiosa (7). El sentido religioso salva asi "toda solución de continuidad con la línea clásica del pensamiento español" (8). O podría pensarse, con más exactitud, que este nuevo espíritu, por prudente y preocupado de no chocar contra la ortodoxia religiosa, trae consigo faialmen:e una liberación respecto al principio de autoridad (particularmente frente a las autori-

5 Labrousse: Essai sur la philosophie politique de l'ancienne Espagne. Paris 1938. p. 231.

6 Menéndez y Pelayo: Historia de los Heterodoxos Españoles. Vol. V, Madrid, 1947, pág. 82.

7 Sánchez Agesta: El pensamiento político del despotismo ilustrado. Instituto de Estudios Políticos. Madrid, 1953. p. 16 ss. y 170 ss.

8 Ibidem, p. 18. 
dades consagradas y sobre todo frente a Aristóteles) que caracteriza el saber medieval (9). Es así como Feijóo, cuyo tradicionalismo se quiere salvar por ser una de las figuras más representativas del siglo, declara que "si la Experiencia y el Evangelio se opusieren, desmentiria mis ojos y mis manos, por asentir al Evangelio" (10) pero igualmente se proclama "ciudadano libre de la República Literaria, ni esclavo de Aristóteles ni aliado de sus enemigos, escucharé siempre con preferencia a toda autoridad privada lo que me dictaren la experiencia y la razón" (11).

Por nuestra parte consideramos fuera de lugar analizar aquí la influencia del desarrollo de la ciencia sobre la filosofía del derecho (12). Nos limitamos a observar que la "nueva fe en la ciencia" no tiene por qué entrar necesariamente en oposición con la fe religiosa, sino que la oposición existe más bien entre el "espíritu geométrico" (para hablar con el lenguaje de Jovellanos) (13) que comportan la nueva ciencia y la filosofía escolástica. Si se parte del supuesto de que todo pensamiento católico es necesariamente fiel a la escolástica medieval, lo que nosotros no aceptamos, esta distinción evidentemente resulta incomprensible.

Primero a través de ese interés por el desarrollo de la ciencia moderna y luego directamente está fuera de duda que la filosofía moderna encuentra acogida en España, a pesar de la Inquisición. Para no citar sino un ejemplo, observemos que inmediatamente después de la expulsión de los jesuitas se emprende una vasta reforma de la enseñanza y GASSENDI, LEIBNITZ, WOLF, etc., se convierten en textos oficiales en las universidades $y$ seminarios. Los ataques a la escolástica, a Aristóteles,

9 Sarrailh: La España Ilustrada de la segunda mitad del siglo XVIII. Tr. Alatorre. FCE. México 1957. p. 441.

10 Feijoo: Teatro Crítico Universal. T. VIII. Disc. VI XXIV. 98.

11 Ibidem. T. IV. disc. XIII. o XI. 35.

Feijoo explica siguiendo al cardenal Pallavicino, que Santo Tomás acogió a Aristóteles para desarmar a los mahometanos y otros enemigos de la Iglesia que se favorecían en su autoridad con Ella. Impugnar a Aristóteles no conducía a nada porque su autoridad estaba establecida entre los árabes. Por eso S. Tomás concibió el proyecto de "traer Aristóteles al bando de la Iglesia”. IV. VI o XII. 34.

12 Sobre esto: Villey: Cours d'histoire de la philosophie $d u$ droit, p. 553 ss y sobre todo p. 577 a 580 . Fasc. V. París 1965 - 1966.

13 "Yo no creo combinables el espíritu geométrico y el escolástico" dice Jovellanos: Carta a Posada. .B A. E. T. L. p. $182 \mathrm{a}$. y las prohibiciones de las controversias y argumentaciones públicas, características del método escolástico, son de ineludible presencia en los proyectos de reforma presentados por las universidades (14).

En cuanto a la filosofía jurídica, hay testimonios aparentemente contrarios. Asi SEMPERE Y GUARINOS se queja: "tenemos en España suma escasez de libros de Derecho Natural y de Gentes. Los principales autores extranjeros que han escrito sobre esta ciencia, Grocio, Puffendorff, Barbeyrac, etc., están prohibidos: nuestros españoles de lo que menos han cuidado ha sido de ella..." y el Derecho Romano bárbaro y afeado se continúa enseñando en las Universidades (15). CADALSO, criticando una primera modificación de la cultura que se vive en la época, se refiere más bien a la excesiva popularidad de esos autores. A diferencia de otras épocas en que no hablaban de esto sino aquellos a quienes competía, como príncipes, embajadores y generales, en ésta - dice - "no hay cadete, estudiante de primer año, ni mancebo de mercader que no hable de Menchaca, Ayala, Grocio, Wolfio, Puffendorff, Vatel, Burlamachy, etc'. (16) Tal vez ambos testimonios sean veraces. Por una parte los mencionados autores modernos no son ni editados ni traducidos en España, salvo excepciones, como puede constatarse en la Biblioteca Nacional de Madrid; por la otra, ellos son citados con complacencia, si no por los mancebos de mercader, al menos por los autores de la época. GARCIA PELAYO explica esta doble actitud por el sentimiento contradictorio que la filosofía jurídica contemporánea inspiraba a los hombres de la época. Por una parte, ellos se esforzaban en construir un Estado fuerte, absolutista; en consecuencia el iusnaturalismo moderno justificación teórica del absolutismo ilustrado, particularmente en su versión austríaca y prusiana, no podrá dejar de seducirlos. Las "alegaciones" de los fiscales del Consejo, por ejemplo, están llenas de citas de obras iusnaturalistas, que utilizan como cantera de argumentos a favor del absolutismo. Pero, por otro lado, no se desconocía el origen protestante y el carácter anticatólico de algunos de sus postulados, proposiciones y consecuencias, lo que tenía que llevarlos a una actitud prudente. "El resultado de esta contraposición hubo de ser el intento de adaptar el lado técnico de tales doctrinas a la concepción del mundo sobre la que reposaba

14 Véase Menéndez y Pelayo: op. cit. Vol. V, pp. 194 ss. 15 Sempere y Guarinos: Ensayo de una Biblioteca española de los mejores escritores del Reynado de Carlos III. Madrid 1873, T. VI. p. 133.

16 Cadalso: Los eruditos a la Violeta. Madrid 1781. p. $32-33$. 
espiritualmente el Estado español, es decir, articular la novedad en la tradición, lo racional en verdades que se estimaban más allá de la razón" (17).

La atracción de los autores modernos es, pues, muy grande por razones politicas y también porque, penetrados ya por el pensamiento de la época, no podian quedarse con una filosofía jurídica cuyas bases estaban destruídas. Es asi como el "método" de estos iusnaturalistas interesa particularmente (18). Las precauciones hacia ellos son igualmente de rigor, porque se los sabe portadores del veneno de la herejía. PIQUER, por ejemplo, dice que para probar ciertas verdades él ha citado autores extranjeros heréticos, pero que no ha estado nunca en su intención conferirles una estima especial; y más adelante observa que cuando los temas que tratan los heréticos "no pertenecen a la religión, sino a la filosofía, medicina y otras ciencias humanas, entonces... se pueden leer y citar con más liberład, bien que es menester, aun en estos casos, mirarlos con cautela, como se disfrazan para ocultar su malicia, y no llenarlos de títulos honoríficos con extremo" (19) MARIN Y M ja, por su parte, "taparse algún tanto los oídos antes de entrar a escuchar las voces de algunos escritores (iusnaturalistas heréticos, porque si no se aventuran a quedar pervertidos con el delicioso encanto de sus pensamientos"' (20). O bien, consultar a los autores católicos que han tratado la materia "puri.icándola de todos sus vicios y señalando la opinión que se debe seguir". Ejemplo de estos autores es JUAN BAUTISTA ALMICI "que habiendo publicado el Derecho Natural y de Gentes de Puffendorf, reformado, dio a luz en Brixia, el año 1768, sus Institutiones Jur. Nat. et Gent. secundum catholica principia, donde en substancia se refunden los elementos de Heineccio..." (21).

En 1770 la cátedra de "Políticas y Económicas, para interpretar las de Aristóteles, ajustando la razón de esta-

17 García Pelayo: prólogo a Marín y Mendoza: Historia del Derecho Natural y de Gentes. Instituto de Estudios Políticos. Madrid, 1950. pp. 7 a 9.

18 Puede verse por ejemplo: Piquer: Philosophia Moral para la juventud española. J. Ibarra. Madrid, 1755. CI o 242. p. 528. Jovellanos recomienda a Vatel como texto para el Derecho de Gentes, por su "método geométrico'’ Plan de Educación de la Nobleza. cit. por García Pelayo. ob. cit. p. 9.

19 Piquer: Discurso sobre la aplicación de la philosophia a los asuntos de religión. Madrid, 1757. pp. 154 y 156. Cit. en Sarrailh: op. cit. 501.

20 Marín y Mendoza: op. cit. p. 59.

21 Ibídem XXXIII pp. 60-61. do con la conciencia, religión y fe católica" (fundada por Felipe IV en 1625 en el Colegio Imperial de Madrid a cargo de los jesuitas "que cuidaron poco de estas cátedras") por la de Derecho Natural y de Gentes (22), indica sin vacilaciones el seniido de la evolución de la filosofía jurídica de la época.

Por nuestra parte no ponemos en duda que haya habido fidelidad religiosa al catolicismo, pero ésta no implicaba un verdadero rechazo de la filosofia moderna. Así las mayores precauciones eran, primero, la de no establecer principios y máximas de Derecho Natural sin saber de antemano si son conformes con la doctrina revelada "y tengan la censura teológica" (23), o la de editar los autores modernos "castigando" su libro según la doctrina católica, es decir, incluyendo refutaciones de las opiniones que parezcan contrarias a la religión. Es eso lo que MARIN Y MENDOZA, primer profesor de la cátedra de Derecho Natural, hizo con el libro de HEINECCIO (24).

En conclusión puede sostenerse que la España del siglo XVIII está profundamente penetrada por la filosofía jurídica de la época y particularmente por la Escuela del Derecho Natural y de Gentes. Que se leyera con preferencia a autores de segundo o tercer rango como AL$\mathrm{MICl} \circ$ que se haya hecho una labor de policía religiosa no tiene para nosotros sino una imporlancia accidenıal. En las páginas que siguen veremos cómo los autores españoles que hemos consultado no sólo son lectores de la nueva filosofia sino que a su vez son constructores de "sistemas" completamente incorporados a la filosofía jurídica de la Escuela del Derecho Natural.

\section{NATURALEZA DE LAS TESIS ESTUDIADAS.}

Para facilitar la lectura expondremos por separado el método de los autores estudiados, su concepto del derecho y el contenido de éste, sabiendo y observando de antemano que los tres aspectos están estrechamente vin-

22 Sempere y Guarinos: op. cit. p. 855.

23 Marín y Mendoza: op. cit. XXXIII. p. 60. "Establecer un principio evidente de Derecho Natural y Político y que en el modo posible indique la sublimidad verdadera de nuestra Sagrada Religión" dice Pérez y López: Principios del orden esencial de la Naturaleza establecido por fundamento de la Moral y Política y por prueba la religión. Nuevo Sistema Filosófico. Madrid 1785. p. XII.

24 Heineccio Elementa iuris Naturae et Gentium, castigationibus ex Catholicorum doctrina et iuris Historia aucta ab Joachino Marin et Mendoza. Madrid 1776. 
culados y que sólo una preocupación de claridad nos lleva a separarlos.

A) EL METODO. - "Yo hallo - dice Piquer (25) que la Philosophia Moral posee algunos principios tan ciertos como la Geometría, pues para mi tan evidente es que no puedo hacer a otro lo que no quiero que se haga conmigo; que no tengo derecho a ofender a nadie; que estoy obligado a querer a mis prójimos; y otras cosas de este modo, como el axioma Geométrico que si a cosas iguales se añaden otras iguales, los todos quedan iguales". Esta ambición de encontrar principios primeros y evidentes sobre el modelo de la geometria, lo comparte Piquer con toda la Escuela del Derecho Natural. Luego, por deducción, se obtiene todo el derecho de esos principios. PEREZ Y LOPEZ también confiesa seguir el método geométrico ( $p$. $X X X V I)$, pero a la vez parece hacer la crítica del racionalismo al denunciar "la llamada razón evidente y el espiritu filosófico" como un mal que corrompe al mundo (p. VII). Ese principio de duda que preconiza la razón moderna es bueno para las verdades físicas, pero no para otras que nuestro espiritu no alcanza en estado de naturaleza caída. El remedio contra ese gravísimo mal no es la radical vuelta a la filosofía medieval, sino el de buscar "un principio de razón tan evidente que disipe los errores... y que en el modo posible indique la sublimidad verdadera de nuestra Sagrada Religión" (p. XII). MARIN Y MENDOZA parece más tradicionalista y denuncia el "escrupuloso delirio" de los que pretendieron derivar todo el derecho natural de un solo y primer principio. Asi critica el método "axiomático" atribuido a Heineccio, el "demostrativo" de Hobbes y el "matemático" de Wolfio (XXXIV a XXXVII. pp. 61 ss.) y hasta hace un moderado elogio del método escolástico (XXXVIII, p. 67); pero esto no le impide estimar que "nadie le puede disputar la gloria (a Wolf) de haber sido el que puso la última mano y el que completó y redujo a perfecto orden y sistema al Derecho de Gentes" (XXII, p. 47) y editar y establecer a Heineccio como texto para sus alumnos. En realidad él parece conforme al método de Heineccio porque propone partir no de uno sino de varios principios $X X X V, p .63$ )y obtener así la certidumbre que es la natural conexión de las verdades de una ciencia con sólidos y claros principios (XXXVII, p. 66). El método axiomático procederia "por distinción y por axiomas o sentencias claras, que se van ordenando una tras otra hasta llegar a sus particulares conclusiones" (XXXVI, pp. $64-65)$.

25 Op. cit. CII. 244.
Evidentemente en el grado de adhesión al método moderno hay diferencias. PIQUER nos parece completamente solidario del racionalismo de la época. "Cuando ocurre una duda sobre la inteligencia de alguna ley civil - dice (CII. 243) - no es preciso recurrir a los Autores y Libros que la interpretan sino al Derecho Natural y de Gentes de donde dimana". MARIN Y MENDOZA, en cambio, observa como un defecto en que incurren los modernos "el desarmar la autoridad, negando la veneración y asenso que se debe a los autores, tanto sagrados como profanos, sobre la suposición que no merecen más fe sus testimonios que en cuanto van conforme con la recta razón... Así, fundado el tirano reino de la razón, ya no consultan, para derivar el Derecho Natural, a los Libros Sagrados; desprecian a los Santos Padres, los teólogos, los escolásticos y jurisconsultos, fiados en una serie de raciocinios que cada cual se esmera en ordenar con más artifício". (XXX, pp. 55-56). Estas protestas, sin embargo, son bien inefectivas y no tienen ningún significado para su obra sobre el Derecho Natural: allí no encontraremos ninguna cita de los Libros Sagrados ni de los Padres de la Iglesia ni de los teólogos y juristas medievales; al contrario, él estima que aun cuando los principios del Derecho Natural se conocieron desde la antigüedad más remota, no bastan los principios sino que había que hallar la relación que tienen entre si y descubrir su ámbito y el arte y preciosa economía de aplicarlos a los casos concretos. Es a comienzos del siglo XVII que se echan, pues, los cimientos de esta nueva ciencia " $y$ entre todos cuantos pueden competir al triunfo de este descubrimiento, sin duda ninguna que se puede contar por el principal a Grocio..." (XII, 30 y 31). Tal vez no valga la pena insistir, esto ilustra suficientemente cuáles son para MARIN las verdaderas autoridades en Derecho Natural.

B) CONCEPTO. - "Por Derecho entendemos el complexo de las leyes que conspiran a un mismo fin", dice PIQUER. La definición no es ningún modelo de rigor, pero ella es, al contrario, un buen signo de la época. En Platón, en San Agustín hay una aproximación del derecho a la ley, pero nunca el derecho, que antes de la época moderna, habia sido definido como un conjunto de leyes. Santo Tomás y los juristas medievales lo definian, al contrario, como aquello que era justo; el derecho no se identifica a las reglas abstractas y generales sino a la solución concreta que se encuentra para el caso. No es el momento de entrar a discutir esa concepción, sino el de observar que al definir al derecho como ley se plantea el delicado problema de elucidar las relaciones entre el derecho y la justicia. PIQUER, en este aspecto, va 
más allá de Grocio y de muchos modernos. Su doctrina, por su rigor y su audacia, puede ser puesta en paralelo con la de Leibnitz, quien tal vez influyó en sus planteamientos. Si el Derecho es el complexo de las leyes, el Derecho Natural "es el conjunto de las leyes generales en toda la universal Naturaleza Humana, en cuanto dictan por la recta razón la conformidad que las acciones del hombre han de tener con el conseguimiento de su verdadera felicidad" (CI. 242). Al Derecho Natural corresponde la justicia universal. La justicia es "la determinación del hombre a executar las acciones libres conforme lo prescribe la ley" (C. 2^0), y la justicia universal corresponde a la ley universalisima de la razón. Esta idea que corresponde a la justicia conmutativa en Leibnitz (expresada en el precepto alterum non laedere) excede por supuesto al Derecho: la justicia universal comprende en sí a todas las virtudes, pues en el ejercicio de ellas el hombre debe conformarse con la recta razón (C. 241).

El Derecho de Gentes es el mismo Derecho Natural aplicado a los hombres en cuanto viven en sociedad (Cl. 242) y el Derecho Civil es el de Gentes en cuanto se acomoda a las diversas circunstancias que concurren en la sociedad humana (Cll. 243). Por la diversidad de circunstancias los príncipes, para gobernar a los pueblos según justicia, se han visto obligados a modificar el Derecho de Gentes acomodando sus máximas a la situación del lugar, del tiempo y de las inclinaciones e intereses de los súbditos (lbid.). Al Derecho Civil corresponde la justicia particular, fundada en esas leyes especiales (C. 240). Pero así como la justicia particular no podria ser contraria a la justicia universal, porque dos virtudes no pueden ser opuestas, el Derecho Civil no puede sino ser conforme al Derecho Natural y de Gentes (CII. 243).

La Ley Divina no es nada diferente de la Ley Natural. Dios, para evitar el error, publicó las leyes del Derecho Natural, que son pocas y pueden reducirse a dos: amar a Dios sobre todas las cosas $y$, segunda, a su prójimo como a si mismo. (Cl. 242).

MARIN $Y$ MENDOZA es menos riguroso pero no menos claro. "Por Derecho Natural - dice (I. p. 16) - se entiende un conjunto de leyes dimanadas de Dios y participadas a los hombres por medio de la razón natural, los cuales teniendo con esta luz bastante auxilio para alcanzar sus más principales preceptos, están obligados, por to mismo, a regular, según ellos, sus acciones“. Observemos que existe en su definición la misma identidad de que parte Piquer: Derecho Natural es el conjunto de leyes naturales. La expresión "natural" tiene una expli- cación que nos parece interesante: "Llámase natural porque no le forman los hombres... (porque lo) lleva el hombre grabado, como dice el Apóstol, en el corazón, desde su cuna, para el gobierno de su vida" (ibídem). No se trata, pues, de un derecho obtenido de la observación de la naturaleza exterior como lo preconizaba Santo Tomás, sino inscrito en el hombre individual, sacado en definitiva de la "naturaleza del hombre". Derecho Natural deviene también en MARIN Derecho Racional. Es por esto que no podemos sino estar en desacuerdo con SANCHEZ AGESTA que quiere representarnos a MARIN como un excelente crítico del Derecho Natural racionalista. (26).

C) CONTENIDO.- Por la naturaleza de sus obras ni PIQUER ni MARIN hablan del contenido del Derecho Natural. PEREZ Y LOPEZ, al contrario, destina todo su libro "Principios..." a tratarlo. El Universo, dice, es el gran Código de la Ley Natural donde están grabados los fines de Dios y de las cosas creadas, "al modo que en un relox están los del artífice y su obra" (p. XIII - XIV). Un inconveniente parece haber sido fácilmente superado: en el mundo y en la naturaleza del hombre hay también corrupción y desorden, por lo que parece arriesgado establecer la naturaleza por principio del derecho natural. La solución de PEREZ Y LOPEZ es sorprendentemente sencilla: admite y demuestra todo lo que es el orden de la naturaleza separándolo del desorden ( $p$. XV). Por esta vía, a través de varias observaciones de la naturaleza, PEREZ Y LOPEZ deduce la inmortalidad del alma, la necesidad de seres racionales en el mundo y la sociabilidad natural del hombre. Estos fines nuestros son a la vez nuestros bienes y en su goce consiste la libertad humana (p. XVIII). "En el supuesto evidente de que Dios ha concedido los bienes y los fines insinuados, y le ha impuesto la obligación de conseguirlos, deduzco: que por necesidad le ha dado derecho, esto es, una facultad moral para adquirir tales bienes y cumplir las referidas obligaciones, y para resistir a los que se opongan a este cumplimiento, pues es imposible que Dios conceda a un hombre derecho para una cosa, y juntamente a otro facultad legítima de impedirsela" (p. XX). Así, por ejemplo, de la necesidad de dar culto a un Dios verdadero se deriva el derecho perfecto para entregarse a ese culto y prohibir la impiedad, la irreligión y la superstición y toda clase de mentira (. XXI). De la necesidad de conservar nuestra vida y salud, el derecho a tener bienes

26 Sánchez Agesta: op. cit. pp. 266 ss. y 291 ss. ("Su obra, como hemos advertido, fue más bien una diatriba contra el Derecho natural racionalista..." (sic) p. 292). 
de fortuna, etc. Así son derivados los derechos de potestad pública, la necesidad de pactos y los derechos más diversos como el de los soberanos a que el Estado y los súbditos tengan religión, derecho a los estudios, a la libre circulación de bienes, etc. Aún cuando el Derecho Natural es necesario, invariable y eterno "y por lo mismo le corresponde en cualquiera situación y tiempo en que se halle" (pp. XXVII-XXVIII), el catálogo de derechos y obligaciones corresponde bien a las aspiraciones y dificultades del fin de la época de Carlos III.

No es tampoco éste el sitio de insistir, pero hoy es bien reconocido que una tendencia común del pensamiento jurídico moderno es la exaltación del derecho subjetivo, concepto que ni Aristóteles ni Santo Tomás, ni los juristas romanos ni medievales conocieron. Tampoco consideramos necesario insistir sobre el paralelismo de las ideas de PEREZ Y LOPEZ con las de WOLFF, que parece haber sido su gran inspirador.

La concepción del método jurídico como una demostración deductiva a partir de primeros principios evidentes, del concepto de derecho como un conjunto de normas que se ordenan en un sistema y el concepto de derecho subjetivo, segunda gran acepción actual del vocablo derecho, nos parecen aspectos absolutamente conexos los unos de los otros, y que nosotros hemos tratado aparte sólo por motivos de claridad. Imposible pensar el método jurídico como una demostración a partir de primeros principios y no concebir el derecho como reglas girando en torno a uno o varios axiomas que fungen de reglas principales, A su vez, si concebimos el derecho como conjunto de reglas de cierta manera inscritas en la naturaleza del hombre, es necesario concluir que el hombre tiene cierias facultades naturales, tan jurídicas como esas leyes naturales y necesarias para cumplir las obligaciones fundamentales que dichas leyes le imponen, como bien lo mostró LOCKE y en España PEREZ $Y$ LOPEZ. Ahora bien, esas tesis corresponden esencialmente a la Escuela del Derecho Natural y de Gentes. En GROCIO, en PUFFENDORFF, en LOCKE y en LEIBNITZ y en una multitud de nombres menores pero que eran muy conocidos en la España del XVIII porque eran escritores de la época, encontramos exactamente las mismas tesis fundamentales.

En la primera parte hemos mostrado cómo los autores modernos y particularmente, en Filosofía del Derecho, los de la Escuela del Derecho Natural y de Gentes, eran conocidos y apreciados en España. En la segunda hemos hablado de las tesis principales de los filósofos juristas escogidos que, asi lo creemos, coinciden con las de la Escuela del Derecho Natural y de Gentes. Obser- vemos ahora que en la Europa moderna existieron otros autores que pertenecieron a corrientes diversas, no identificables a la Escuela del Derecho Natural, que no fueron ni apreciados ni seguidos por los autores estudiados. Es el caso de HOBBES y SPINOZA y más tarde el de ROUSSEAU y MONTESQUIEU, que aunque familiares a dicha escuela, introducen novedades notables. Para esquematizar resumamos estas doctrinas en dos grupos: el positivismo voluntarista, que reduce el derecho a la voluntad del Estado y el naturalismo jurídico, que insiste en la dependencia del derecho de factores naturales, tales como el clima, la latitud o la raza. ¿Por qué Piquer, Marín y Pérez y López, representantes para nosotros de la filosofía jurídica del XVIII español, han sido hostiles o ignorado esas nuevas corrientes de pensamiento? La respuesta a esa pregunta, la razón de esa selección, naturalmente no expresada en las obras consultadas, me parece bien compleja de encontrar. En esta conclusión nos ocuparemos de ellos sin pretender más que una aproximación, pues el punto nos lleva a complicados problemas de filiación filosófica que hemos mencionado en la introducción.

La separación tajante de la historia de la filosofía juridica por épocas, es, al parecer, una fuente de equívocos. No es que no existan tales separaciones sino que los criterios de distinción deben ser constantemente revisados, deshechos y rehechos, como una tela de Penélope, y que conviene insistir tanto en los puntos de separación como los de contacto. Así se ha dicho que el Derecho Natural moderno es laico y racionalista, por oposición a las doctrinas medievales, fundadas sobre la Revelación; $y$ ese contraste ha sido aceptado pasivamente por los que se han ocupado de la historia de la filosofía del derecho en España. Contemporáneamente muchos historiadores insisten, por el contrario, sobre el carácter laico de la filosofía juridica tomista y sobre el lugar preponderante que ésta concede a la razón (27). La realidad parece, pues, más matizada. Estas investigaciones recientes han mostrado suficientemente las relaciones, los puentes, entre el pensamiento jurídico medieval y el moderno. El fuerte acento que pone Santo Tomás en las posibilidades naturales del hombre de aprehender, por observación, elementos jurídicos, y el lugar, relativamente redu-

27 V. Villey: Consideraciones en pro del Derecho Natural Clásico. Tr. Garzón Valdéz. Universidad de Córdoba. Argentina, 1966 .Leçons d'Histoire de la Philosophie du Droit, pp. 109 ss, 203 ss. Sobre el "tradicionalismo" del Derecho Natural moderno del mismo autor: Les fondateurs de l'école du droit naturel moderne. Archives de Philosophie du droit, 1961. pp. 73-105. 
cido en relación al agustinismo jurídico, que confiere a la Revelación en el derecho, lo hace sin duda un antecedente importantísimo de la Escuela del Derecho $\mathrm{Na}$ tural y de Gentes. La escolástica española del siglo XVI sirvió justamente de intermediaria entre ambos. Por lo esencial, las ideas de Grocio se encuentran ya en Suárez. Ambos autores hacen una síntesis de la doctrina tomista con el nominalismo occamiano que llegó a dominar en las universidades europeas en el siglo XV. Santo Tomás, Suárez y Grocio tienen confianza en la razón y tienden al laicismo, en oposición a la línea agustinista que retoma Occam. Que sea una razón diferente la de Santo Tomás a la de Grocio es, a nuestra manera de ver, una verdad indiscutible, pero que por el momento podemos dejar de lado. Marín y Mendoza no se equivoca al referirse a Vitoria, Soto, Vásquez de Menchaca y "otros sabios españoles" (lo que sin duda incluye a Suárez, Molina, Mariana, etc., prohibidos para la época) como importantes en la formación del pensamiento grociano (28).

Estaria, por supuesto, lejos de nuestra intención discutir el carácter moderno de la Escuela del Derecho Natural y de Gentes. Ella se halla suficientemente penetrada por el nominalismo, que es otra doctrina medieval, pero que domina toda la modernidad, y por el neoestoicismo, para hacer cambiar de sentido los elementos que toma del tomismo. Así ya no será la razón realista, que parte de conjuntos y que pretende llegar sólo a verdades probables (salvo, en Santo Tomás, confirmación de la Escritura Santa en determinadas materias) sino la rigurosa lógica de los nominalistas, que procede por análisis o en todo caso a partir de verdades claras y evidentes, es decir de verdaderos átomos lógicos, y que conduce deductivamente todo el discurso hasta llegar a conclusiones particulares y seguras. Vitoria y Suárez fueron también sensibles a estas influencias. Sostener que su

28 Marín y Mendoza: op. cit. XI. p. 29. pensamiento es absolutamente fiel al tomismo sería desconocer el papel clave que tienen en la formación del pensamiento moderno y todo el interés que tienen sus doctrinas.

Esta continuidad entre el iusnaturalismo moderno con la Escolástica española lo hizo atractivo y fácilmente aceptable por los autores españoles del XVIII. Tal vez, igualmente, buena parte de los neotomismas del XIX y aún del $X X$, no entronquen con Santo Tomás sino a través de Wolff y Leibnitz y Suárez. Es en esa tradición donde se inscriben los autores españoles del XVIII que hemos estudiado. Ellos son tradicionalistas en otro aspecto, en la medida en que permanecen hostiles a corrientes mucho más novedosas que alcanzan éxito en la Europa del siglo XVIII, como el voluntarismo y el naturalismo mencionados, que constituyen las dos grandes ramas del positivismo jurídico. Es justamente su tradición racionalista, su filiación, en definitiva algo bastarda, con el pensamiento de Santo Tomás, lo que imposibilita que simpaticen con estas corrientes que son igualmente modernas, pero que tal vez, dentro de la modernidad, más revolucionarias desde el punto de vista de la filosofía jurídica.

Dejemos claro, para terminar, que no afirmamos que en España, en el XVIII, se desconociera a Hobbes o a Rousseau. Marin y Mendoza les da a cada uno un sitio especial en los comentarios que dedica a los autores, y aun Feijóo llegó a leer las primeras obras de Rousseau. Es más, en ciertas obras de política o de economía, como las de CAMPOMANES, CABARRUS o en el famoso Informe sobre la Reforma Agraria de JOVELLANOS, es ya notoria y decisiva la influencia de Rousseau o de los fisiócratas. Pero estas obras no conciernen directamente, a nuestra manera de ver, la filosofía del derecho. Será otra época, los comienzos del siglo XIX, con Martínez Marina y Alcalá Galiano, la que incorporará estos autores a la historia de la filosofía jurídica en España. 